EPJ Web of Conferences 95,01010 (2015)

DOI: 10.1051 /epjconf/20159501010

(C) Owned by the authors, published by EDP Sciences, 2015

\title{
Large-scale shell-model study of the Sn isotopes
}

\author{
Eivind Osnes ${ }^{1 \mathrm{a}}$, Torgeir Engeland ${ }^{1}$, and Morten Hjorth-Jensen ${ }^{1,2}$ \\ ${ }^{1}$ Department of Physics, University of Oslo,N-0316 Oslo, Norway \\ ${ }^{2}$ National Superconducting Cyclotron Laboratory, Department of Physics and Astronomy, Michigan State University, \\ East Lansing, MI 48824, USA
}

\begin{abstract}
We summarize the results of an extensive study of the structure of the Sn isotopes using a large shell-model space and effective interactions evaluated from realistic two-nucleon potentials. For a fuller account, see ref. [1].
\end{abstract}

\section{Introduction}

In the $\mathrm{Sn}$ region the number of single-particle degrees of freedom is so high that ab initio approaches are prohibited. Thus, the most 'fundamental' approach which can be applied is the shell model - the 'work horse' of nuclear structure calculations. Still, using ${ }^{100} \mathrm{Sn}$ as closed-shell core and allowing the valence neutrons to occupy the $2 s 1 d$ shell plus the intruder orbits $0 g_{7 / 2}$ from below and $0 h_{11 / 2}$ from above, one need cope with very high dimensionality. The maximum number of basis states is obtained mid-shell and amounts to more than 16 million in the $m$-scheme. To deal with such high dimensionality we resort to the Lanczos algorithm. As there are 32 distinct single-particle $m$-states in the chosen valence space, it is convenient to represent the different basis states with a 32-bit computer word, with 0's and 1's for unoccupied and occupied single-particle states, respectively. Then, applying operators to such states amounts to moving the 0's and 1's about.

From a physical point of view, the $\mathrm{Sn}$ isotopes are particularly interesting, as the excitation energy of the first $2^{+}$state is nearly constant throughout the entire region from ${ }^{102} \mathrm{Sn}$ to ${ }^{130} \mathrm{Sn}$. This is indicative of the validity of the general seniority coupling scheme [2]. Further, it is of interest to study how the excitation spectra develop over this extended region of isotopes between the ${ }^{100} \mathrm{Sn}$ and ${ }^{132} \mathrm{Sn}$ closed shells.

In the early years of the shell model one would only have sufficient computer power to study nuclei near closed shells. However, in Sn those nuclei are unstable and were not accessible to experimental study at the time. Only the nuclei in the middle of the shell were stable, but these were inaccessible to shell-model calculations and could only be studied by cruder approximations such as the BCS and RPA and using very phenomenological interactions [3]. Today, on the one hand, adequate computer power is available for handling shell-model calculations in the mid-shell Sn isotopes, and on the other hand, the unstable isotopes near the closed shells are accessible to experimental study. Thus, one is now able to study the whole string of $\mathrm{Sn}$ isotopes from ${ }^{100} \mathrm{Sn}$ to ${ }^{132} \mathrm{Sn}$ on the same footing using the shell model.

\footnotetext{
ae-mail: eivind.osnes@fys.uio.no
} 


\section{Effective interaction}

A crucial question for shell-model calculations is which effective interaction to use. Our aim is to use effective interactions calculated from realistic two-nucleon forces (i.e., reproducing the deuteron binding energy and two-nucleon scattering phase shifts) using many-body perturbation theory. In the present work we have compared effective interactions obtained from three different nucleon-nucleon potentials, viz. the Argonne V18 [4], the CD Bonn [5] and the chiral N3LO [6] potentials. In obtaining the effective interaction from these potentials we have used the $Q$-box approach of Kuo and collaborators [7]. The so-called $Q$-box is the sum of all irreducible valence-linked diagrams. The $Q$ boxes are then folded into each other an arbitrary number of times. In the first step of the calculation of the $Q$-box one has to eliminate the strong short-range repulsion by some sort of regularization procedure. For comparison we have applied both the $G$-matrix and the $V_{\text {lowk }}$ interactions as our firstorder input in the $Q$-box calculation. The $Q$-box is evaluated to third order in these interactions. We also compare $Q$-boxes evaluated with intermediate excitations varying from $2 \hbar \omega$ to $10 \hbar \omega$ harmonic oscillator energy.

\section{Explorative calculations}

In a first explorative study we considered the even isotopes from ${ }^{102} \mathrm{Sn}$ to ${ }^{116} \mathrm{Sn}$ and compared the results obtained for the three different nucleon-nucleon potentials, starting from both the $G$-matrix and the $V_{\text {lowk }}$ interactions, and using $Q$-boxes including up to $2 \hbar \omega, 4 \hbar \omega, \ldots, 10 \hbar \omega$ harmonic oscillator excitation energies in the intermediate states. The essential findings of these explorative calculations may be summarized as follows:

1) All energy calculations are reasonably well converged by including up to $10 \hbar \omega$ intermediate excitation energy in the $Q$-box.

2) All potentials gave nearly constant $2^{+}$spacing throughout the isotopes. For $V_{\text {lowk }}$ the three potentials gave very little difference in the $2^{+}$energy gap, but this was much larger than the measured gap. Better agreement was obtained with the $G$-matrix, but here the difference between the potentials was larger than for $V_{\text {lowk }}$. Also for the $G$-matrix all three potentials gave too large $2^{+}$spacing. The V18 potential gave the smallest spacing, the CD Bonn the largest one.

3) The ground-state binding energies with respect to ${ }^{100} \mathrm{Sn}$ were roughly reproduced for the lowest $\mathrm{Sn}$ isotopes. However, from ${ }^{108} \mathrm{Sn}$ on increasing overbinding was obtained. Again, the three potentials gave similar binding energies for $V_{\text {lowk }}$, but stronger overbinding than for the G-matrix, in which case the V18 potential gave the strongest overbinding. The binding energy calculations demonstrated clearly that it is necessary to include high intermediate excitations in the $Q$-box.

\section{Final calculations}

Then, final extensive calculations of all the isotopes from ${ }^{102} \mathrm{Sn}$ to ${ }^{132} \mathrm{Sn}$ were performed using a $G$ matrix based third-order $Q$-box calculated from the chiral N3LO nucleon-nucleon potential and including intermediate excitations up to $10 \hbar \omega$ harmonic oscillator energy. The nuclear properties evaluated were the ground-state binding energies with respect to the ${ }^{100} \mathrm{Sn}$ closed-shell core, separation energies, excitation energies, and $\mathrm{B}(\mathrm{E} 2)$ values for the transition $0_{\mathrm{gs}}^{+} \rightarrow 2^{+}{ }_{1}$.

The results of the full calculation confirm the trends shown by the explorative calculations summarized above. The near constancy of the $2^{+}$and $4^{+}$spacings with respect to the ground state is well reproduced by the calculations, although the $2^{+}-0^{+}$spacing is on average $250 \mathrm{keV}$ too big, reflecting that the pairing component of the effective interaction is too large. The ground-state binding 
energies agree well for the isotopes with only a few valence nucleons, while increasing overbinding occurs from ${ }^{108} \mathrm{Sn}$ on. This may be corrected by adding a modest average repulsion to the interaction. This could come from effective three-body forces. It is instructive to consider the one-particle separation energies, which are the binding energy differences between neighboring nuclei. We then get separate curves for the even and odd isotopes. Each curve is nearly linear, being displaced by the pairing energy. This indicates that we may approximate the binding energy as a function of the number $n$ of valence nucleons by [8]

$$
E(n)=n \varepsilon+1 / 2 n(n-1) \alpha+\left[\frac{n}{2}\right] \beta,
$$

where $\varepsilon$ represents the binding energy of a single valence nucleon to the core, and $\alpha$ and $\beta$ are average two-nucleon interaction parameters. The notation $[n / 2]$ means the largest integer not exceeding $n / 2$. Thus, $\beta$ is the average pairing energy, whereas $\alpha$ is the slope of the separation energy curves. From empirical fits to typical shell-model nuclei in different regions of the nuclear chart one finds that $\beta$ is typically large and attractive, and $\alpha$ is small and repulsive. However, the value of $\alpha$ extracted from our calculation is too small, reflecting insufficient average repulsion of our effective interaction. This is in fact a typical features of most realistic effective interactions calculated to date. The B(E2) rates agree well for the heavy isotopes, while they seem to be too low for the light isotopes. This discrepancy cannot be overcome in a consistent way by increasing the effective charges, since this would destroy the agreement for the heavier isotopes. A possible solution to this may be to consider proton excitations out of the ${ }^{100} \mathrm{Sn}$ core. On the other hand, the measured $\mathrm{B}(\mathrm{E} 2)$ rates of the light isotopes [9] have rather large error bars, and their accuracy would need to be improved before firm conclusions can be drawn.

\section{Summary and outlook}

In summary several remarks may be made:

4) It is remarkable that an effective interaction calculated from a basic realistic nucleon-nucleon interaction using sophisticated many-particle perturbation methods is capable of giving not only qualitative, but also to some extent quantitative reproduction of nuclear data over an extensive range of nuclei involving a large number of valence nucleons.

5) Still, it is a challenge to improve the effective interaction in order to obtain sufficient average repulsion. Including effective three-body terms may be a way out, but this represent a rather complicated calculation.

6) Another challenge is to see which effect proton excitations out of the ${ }^{100} \mathrm{Sn}$ core would have on the B(E2) rates of the light $\mathrm{Sn}$ isotopes. Such a calculation is rather involved, so it may be useful first to consider analogous but lighter nuclear systems, such as the Ni isotopes beyond ${ }^{56} \mathrm{Ni}$.

7) From the experimental side we would like to see more accurate measurements of the $\mathrm{B}(\mathrm{E} 2)$ rates in the light isotopes, as well as more precise mass (binding energy) measurements in the very low-mass $\mathrm{Sn}$ isotopes.

\section{References}

1. T. Engeland, M. Hjorth-Jensen, M. Kartamyshev, E. Osnes, Nucl. Phys. A 928, 51 (2014)

2. I. Talmi, Nucl. Phys. A 172, 1 (1971)

3. L.S. Kisslinger, R.A. Sorensen, Fys. Medd. Dan. Vid. Selsk. 32, 1 (1960); 
T.T.S. Kuo, E.U. Baranger, M. Baranger, Nucl. Phys. 79, 513 (1966)

4. R.B. Wiringa, V.G.J. Stocks, R. Schiavilla, Phys. Rev C 51, 38 (1995)

5. R. Machleidt, et al., Phys. Rev. C 63, 024001 (2001)

6. D.R. Entem, et al., Phys. Rev C 68, 041001 (2003)

7. T.T.S. Kuo, E. Osnes, Folded-Diagram Theory of the Effective Interaction in Atomic Nuclei, Springer Lecture Notes in Physics, vol. 364, Springer, Berlin (1990), and references therein; M. Hjorth-Jensen, T.T.S. Kuo, E. Osnes, Phys. Rep. 261, 125 (1995)

8. A. De-Shalit, I. Talmi, Nuclear Shell Theory, Academic Press, New York (1963), p. 342ff

9. A. Banu, et al., Phys. Rev. C 72, 061305(R) (2005);

A. Ekström, et. Al., Phys. Rev. Lett. 101, 012502 (2008);

V.M. Bader, et al., Rhys. Rev. C 88, 051301(R) (2013). 